\title{
Lymph Node Assessment: Quality, Not Quantity
}

\author{
Anand Govindarajan, MD, MSc ${ }^{1,3}$ and Nancy N. Baxter, MD, $\mathbf{P h D}^{2,3}$ \\ ${ }^{1}$ Department of Surgery, Mount Sinai Hospital, University of Toronto, Toronto, Canada; ${ }^{2}$ Department of Surgery and \\ Institute of Health Policy, Management and Evaluation, Li Ka Shing Knowledge Institute, St. Michael's Hospital, \\ University of Toronto, Toronto, ON, Canada; ${ }^{3}$ Institute for Clinical Evaluative Sciences, Toronto, Canada
}

Adequate lymph node staging in gastrointestinal cancers is a key component of quality cancer care as staging has major prognostic relevance and potential implications for adjuvant therapy. What is controversial, however, is the exact method and the metrics that will optimize nodal staging. One issue that is frequently studied is the extent of lymphadenectomy.

Extent of lymphadenectomy has been studied from the perspectives of the impact of the number of lymph nodes in a standard lymphadenectomy and the impact of extended versus standard lymphadenectomy. While most studies in the Western literature have not shown a significant benefit to extended lymphadenectomy for most disease sites, the converse is true for the number of lymph nodes. ${ }^{1,2}$ Myriad studies in most disease sites demonstrate that survival is significantly associated with lymph node number, particularly in node-negative disease. ${ }^{3}$ Sampling and stage migration are frequently cited as the primary mechanism for this relationship. This has translated into recommended minimums being codified in current guidelines for colorectal cancer (12 nodes), gastric cancer (16 nodes), and esophageal cancer (15 nodes) among others. More importantly, some of these thresholds have been adopted as quality metrics. ${ }^{4}$ However, it is important to recognize that

Dr. Baxter holds the Cancer Care Ontario Health Services Research. The Institute for Clinical Evaluative Sciences is funded by an annual grant from the Ontario Ministry of Health and Long-Term Care. The opinions reported in this paper are those of the authors and are independent from the funding sources. No endorsement by the Institute for Clinical Evaluative Sciences or the Ontario Ministry of Health and Long-Term Care is intended or should be inferred.

(C) Society of Surgical Oncology 2013

Published Online: 18 May 2013

N. N. Baxter, MD, PhD

e-mail: baxtern@smh.toronto.on.ca the relationship between the number of lymph nodes evaluated and improved oncologic outcomes represents association, not causation. Several factors other than understaging may explain this association, including the presence of confounding variables related to patient factors and the patient-tumor immunologic response. ${ }^{5-7}$ The latter theory implies that the number of assessable lymph nodes may be related, at least in part, to tumor biology, and thus the absolute number of lymph nodes assessed may not always be a marker of quality.

The current standard of 12 nodes for colorectal cancer is based largely on data from colon cancer and extrapolated to rectal cancer. However, the current paradigm of neoadjuvant chemoradiation for the treatment of rectal cancer complicates this issue because radiation is known to result in a decrease in the number of assessable lymph nodes. ${ }^{8}$ Currently, we do not fully understand the possible implications, or lack thereof, of this decreased nodal harvest. We have previously demonstrated that although the total number of nodes assessable is lower in rectal cancer patients who have undergone preoperative neoadjuvant chemoradiation, there is no clinical impact on nodal staging or oncologic outcome. ${ }^{9}$ In this issue of the Annals, de Campos-Lobato and colleagues present work that adds to our understanding of this issue. ${ }^{10}$ In their examination of 237 patients treated with long-course neoadjuvant chemoradiation for locally advanced rectal cancer (clinical stage II and III), they similarly found no significant association between number of nodes assessed (dichotomized as $<12$ vs. $>12$ ) and most oncologic outcomes, with the exception of local recurrence. However, they did find a significant association between final pathologic stage and number of lymph nodes assessed: more patients with a complete pathologic response had less than 12 nodes assessed (36 vs. $19 \%)$. This supports the concept that the number of assessable lymph nodes reflects phenomena related to 
tumor biology - the greater the tumor response to radiation, the greater the reduction in the number of assessable nodes. Indeed, this likely explains the significantly lower local recurrence rates in the group where $<12$ nodes were assessed.

The authors also touch on a second consideration in nodal staging - the best metric to use to reflect nodal status. The absolute number of positive nodes is the traditional measure used and is incorporated into current TNM staging systems, with more positive lymph nodes generally portending a worse prognosis. Recently, lymph node ratio (LNR, the ratio of positive to total lymph nodes) has been studied and put forward as a better metric of nodal staging based on potentially improved prognostic accuracy..$^{11,12}$ However, it is difficult to assess its relative benefit based on the current study as the LNR was calculated for the entire study population, rather than just the node-positive patients where it would be most relevant; therefore, its strong association with outcome in the present study may just reflect the prognostic value of node-positive versus nodenegative status. In addition, clinically important LNR thresholds that are validated across multiple study populations have yet to be defined. Therefore, LNR is an intriguing concept that is worthy of more study but is not yet ready for mainstream use.

What can we take away from this study and other emerging data in this area? although it can be difficult to assess 12 lymph nodes in rectal cancer patients treated with neoadjuvant chemoradiation, a low lymph node count is unlikely to result in understaging and the nodal status postchemoradiation can be considered accurate. It is unlikely that expensive fat-clearing techniques to attempt to find more lymph nodes for these patients will "improve" staging accuracy in this setting.

Notably, this work does not obviate the importance of the quality of surgical technique (e.g., sharp mesorectal excision) and pathological assessment, as pillars of accurate staging. Poor quality surgical or pathological processes will lead to inaccurate staging, especially in the postchemoradiation setting where nodes can be more difficult to find. Indeed, quality initiatives alone may improve lymph node assessment and potentially improve staging. ${ }^{13}$

However, if these processes are optimized, the number of lymph nodes assessed is not a reflection of quality but rather tumor biology. Therefore, we must continue to focus on improving the surgical and pathological processes of care to optimize lymph node assessment, rather than focusing on arbitrary outcomes, such as achieving a minimum number of lymph nodes as a marker of quality.

\section{REFERENCES}

1. Riall TS, Cameron JL, Lillemoe KD, Campbell KA, Sauter PK, Coleman J, et al. Pancreaticoduodenectomy with or without distal gastrectomy and extended retroperitoneal lymphadenectomy for periampullary adenocarcinoma-part 3: update on 5-year survival. J Gastrointest Surg. 2005;9(9):1191-204; discussion 1204.

2. Georgiou P, Tan E, Gouvas N, Antoniou A, Brown G, Nicholls $\mathrm{RJ}$, et al. Extended lymphadenectomy versus conventional surgery for rectal cancer: a meta-analysis. Lancet Oncol. 2009; 10(11):1053-62.

3. Chang GJ, Rodriguez-Bigas MA, Skibber JM, Moyer VA. Lymph node evaluation and survival after curative resection of colon cancer: systematic review. J Natl Cancer Inst. 2007;99(6): 433-41.

4. Desch CE, McNiff KK, Schneider EC, Schrag D, McClure J, Lepisto E, et al. American Society of Clinical Oncology/National Comprehensive Cancer Network Quality Measures. J Clin Oncol. 2008;26(21):3631-7.

5. Govindarajan A, Baxter NN. Lymph node evaluation in earlystage colon cancer. Clin Colorectal Cancer. 2008;7(4):240-6.

6. George S, Primrose J, Talbot R, Smith J, Mullee M, Bailey D, et al. Will Rogers revisited: prospective observational study of survival of 3592 patients with colorectal cancer according to number of nodes examined by pathologists. $\mathrm{Br} J$ Cancer. 2006;95(7):841-7.

7. Leibl S, Tsybrovskyy O, Denk H. How many lymph nodes are necessary to stage early and advanced adenocarcinoma of the sigmoid colon and upper rectum? Virchows Arch. 2003;443(2): 133-8.

8. Baxter NN, Morris AM, Rothenberger DA, Tepper JE. Impact of preoperative radiation for rectal cancer on subsequent lymph node evaluation: a population-based analysis. Int J Radiat Oncol Biol Phys. 2005;61(2):426-31.

9. Govindarajan A, Gönen M, Weiser MR, Shia J, Temple LK, Guillem JG, et al. Challenging the feasibility and clinical significance of current guidelines on lymph node examination in rectal cancer in the era of neoadjuvant therapy. J Clin Oncol. 2011;29(34):4568-73.

10. de Campos-Lobato LF, Stocchi L, de Sousa JB, Buta M, Lavery IC, Fazio VW, et al. Less than 12 nodes in the surgical specimen after total mesorectal excision following neoadjuvant chemoradiation. Ann Surg Oncol. 2013. doi:10.1245/s10434-013-3010-x.

11. Ceelen W, Van Nieuwenhove Y, Pattyn P. Prognostic value of the lymph node ratio in stage III colorectal cancer: a systematic review. Ann Surg Oncol. 2010;17(11):2847-55.

12. Wang LP, Wang HY, Cao R, Zhu C, Wu XZ. Proposal of a new classification for stage III colorectal cancer based on the number and ratio of metastatic lymph nodes. World J Surg. 2013;37(5): 1094-102.

13. Smith AJ, Law CHL, Khalifa MA, Hsieh ETK, Hanna SS, Wright $\mathrm{FC}$, et al. Multimodal CME for surgeons and pathologists improves colon cancer staging. J Cancer Educ. 2003;18(2):81-6. 